OPEN ACCESS

Edited by:

Silvia Carloni,

University of Urbino Carlo Bo, Italy

Reviewed by:

Anna Tarocco,

University of Ferrara, Italy

Qianshen Zhang,

University of Hong Kong, China

*Correspondence:

Yuan Shi

shiyuan@hospital.cqmu.edu.cn

Specialty section:

This article was submitted to

Neonatology,

a section of the journal

Frontiers in Pediatrics

Received: 01 March 2020

Accepted: 15 June 2020

Published: 23 July 2020

Citation:

MaX, Wu X and Shi Y (2020) Changes

of Dynamic Functional Connectivity Associated With Maturity in Late

Preterm Infants. Front. Pediatr. 8:412.

doi: 10.3389/fped.2020.00412

\section{Changes of Dynamic Functional Connectivity Associated With Maturity in Late Preterm Infants}

\author{
Xueling Ma ${ }^{1,2,3}$, Xiushuang $W u^{4,5}$ and Yuan Shi ${ }^{1,2,3,6 *}$ \\ ${ }^{1}$ Department of Neonatalogy, Children's Hospital of Chongqing Medical University, Chongqing, China, ${ }^{2}$ National Clinical \\ Research Center for Child Health and Disorders, Chongqing, China, ${ }^{3}$ Key Laboratory of Child Development and Disorders, \\ Ministry of Education, Chongqing, China, ${ }^{4}$ Department of Pediatrics, Daping Hospital, Army Medical University, Chongqing, \\ China, ${ }^{5}$ Department of Pediatrics, Yunnan Provincial Crops of Chinese People's Armed Police Force, Langfang, China, \\ ${ }^{6}$ Chongqing Key Laboratory of Child Infection and Immunity, Chongqing, China
}

Objective: To investigate the changes of dynamic functional connectivity (DFC) in late preterm infants, and assess whether these changes are associated with the indicators measuring the maturity of neonates.

Methods: Resting-state fMRI (rs-fMRI) data of eligible neonates was acquired with a 3.0-T MRI scanner in the Department of Radiology, Daping Hospital, Army Medical University (Chongqing, China). After the selection of functional connectivity networks obtained by independent component analysis (ICA), a sliding-window approach was used to cluster all the windows into different states. Then the differences of temporal properties of DFC between groups were compared, and the association between these temporal properties and the degree of maturity was also explored in each state.

Results: Eventually, 34 late preterm and 37 term neonates were included in the final analysis. Based on their data, 5 components were located in 5 networks: default-mode (DMN), dorsal attention (DAN), auditory (AUD), sensorimotor (SMN), and visual (VN). Then four reoccurring state patterns of functional connectivity were identified with the k-means clustering method. The late preterm group dwelled significantly longer in State III (late preterm: $33.57 \pm 37.64 \mathrm{~s}$, term: $18.50 \pm 11.71 \mathrm{~s} ; P=0.03)$, which was characterized by general weaker connectivity between networks. Also, the correlation analysis shows the degree of maturity is negatively correlated to the dwell time and fractional windows in State III.

Conclusion: Our findings suggested that compared with term infants, late preterm infants preferred to stay in a state with general weak connectivity between networks, but this preference declined as maturity increased.

Keywords: late preterm infants, premature brain injury, fMRI, independent component analysis, dynamic functional connectivity 


\section{INTRODUCTION}

Preterm birth accounts for $11 \%$ of all live-births worldwide, and their survival rate has remarkably increased in recent few years $(1,2)$. Preterm infants, even late preterm infants (born between $34^{+0}$ and $36^{+6}$ weeks of gestation), are still at risk for neurodevelopmental impairment. Accounting for about $75 \%$ of all preterm births, late preterm infants are the largest group of preterm newborns. Severe brain injuries such as cerebral palsy seldom happen in late preterm infants, but mild to moderate injuries often occur in this group. According to recent studies, mild injuries may also lead to developmental and emotional-behavioral problems in adolescence, involving language disorder, attention deficit hyperactivity disorder, developmental coordination disorder, autism spectrum disorder, and so on $(3,4)$.

A series of characteristic alterations in the structural and functional connectivity of brain were identified in many neurological disorders. By observing these alterations, the associations across many brain disorders may be revealed (5). As for neurodevelopmental problems caused by premature birth, both commonalities and differences in clinical characteristics have been noticed. Therefore, exploring characteristic alterations of the connectivity networks in preterm infants may help reveal mechanisms of various disorders related to premature brain injury. For instance, lesions in different locations can cause similar symptoms if these lesions affect the same brain network. For this reason, we chose to analyze the functional network connectivity in late preterm neonates.

A recent technique called dynamic functional connectivity (DFC), assesses temporal variations of functional connectivity during MRI acquisition by dividing resting-state functional MRI (rs-fMRI) scans into a series of "sliding windows" $(6,7)$ and clustering these windows into several states by k-means method $(6,8)$.

We hypothesized that the temporal properties of DFC between term and late preterm infants were different, and there were associations between these properties and indicators measuring maturity.

\section{MATERIALS AND METHODS}

\section{Participants}

A total of 155 neonates were initially recruited from the Department of Pediatrics, Daping Hospital, Army Medical University (Chongqing, China), including 58 term and 97 late preterm infants. Enrolled patients were initially chosen following these criteria: neonates without unstable medical condition or contraindication to MRI, gestational age more than 34 weeks, no acute or chronic diseases, no resuscitation history at birth, no major congenital malformations, and no congenital infections. After the acquisition of MRI data, two experienced experts reviewed the imaging and reported the result together, infants with definite or suspicious intracranial hemorrhage or other major structural abnormalities were also excluded in the following analysis. This step excluded 32 preterm and 3 term infants, remaining 120 infants in the dataset.
This study was approved by the Ethics Committee of Daping Hospital, Army Medical University (Chongqing, China). All study procedures followed the Declaration of Helsinki. Written informed consent was obtained from every infant's parents.

\section{Data Acquisition}

Scans were collected using a 3.0-T MRI scanner (Siemens, Germany) during natural sleep in the Department of Radiology, Daping Hospital, Army Medical University (Chongqing, China). Infants were transported to MRI scanner, accompanied by a nurse and a neonatologist. Scanning was immediately done after the infant was fed to induce drowsiness. Neonatal ear muffs were used to block out MRI noise. During the examination, each participant was continuously monitored by an electrocardiogram and a pulse oximeter and closely observed by the accompanied neonatologist. Structural images were collected with a turbo-spin echo (TSE), T2-weighted sequence, TR/TE/flip angle $=3,200 / 393$ $\mathrm{ms} / 150^{\circ}$, voxel size $=1.25 \times 1.25 \times 1.95 \mathrm{~mm}^{3} ; 96$ transversal slices, bandwidth $=751 \mathrm{~Hz} /$ pixel. Rs-fMRI scans were collected using a T2-weighted echo-planar imaging (EPI) sequence, field of view $=220 \times 220 \mathrm{~mm}^{2}, \mathrm{TR} / \mathrm{TE} / \mathrm{flip}$ angle $=2,000 / 30 \mathrm{~ms} / 90^{\circ}$, voxel size $=3.4 \times 3.4 \times 3.0 \mathrm{~mm}^{3}$ with no gap, number of slices $=33$. For each neonate, 240 volumes were obtained across the whole brain.

\section{Preprocessing}

The rs-fMRI data was preprocessed using Gretna2.0 (9) implemented in MATLAB (version R2013b)/SPM12. At the beginning of preprocessing, the first 10 volumes were removed to reach a steady state, leaving 230 volumes for each infant. Slice timing (middle slice as reference slice) was carried out for correction of acquisition time delay between slices, and realignment (register to mean) was carried out for correction of head motion between volumes. Since excessive head motion can affect DFC analysis (10), conservative inclusion criteria were chosen to minimize head-motion bias, which means acquisitions with frame wise displacement $(\mathrm{FD})>0.5 \mathrm{~mm}$ would be removed (11), as well as the ones with translational movement more than $2 \mathrm{~mm}$ or rotational movement more than $2^{\circ}$. According to these criteria, we excluded 31 late preterm infants and 18 term infants, and 34 late preterm and 37 term infants were included in the final analysis. The following steps included reorientation manually, spatial normalization with EPI template (12), spatially smoothing with a Gaussian kernel (full width at half-maximum of $4 \mathrm{~mm}$ ). The flow chart of data processing is shown in Figure 1.

\section{Group Independent Component Analysis}

After data preprocessing, a data-driven technique called spatial independent component analysis (ICA) was performed with GIFT (version 3.0b) $(13,14)$ to decompose the data into spatial independent components. Two steps for data reductions were run during the analysis, that is, the subject-level and grouplevel principal component analysis. ICA was performed under the component number from 14 to 30 to obtain stable infant networks (the number estimated by minimum description length criteria is 14), and at last the component number of 25 was chosen for its relatively stable and intact network profiles. To 


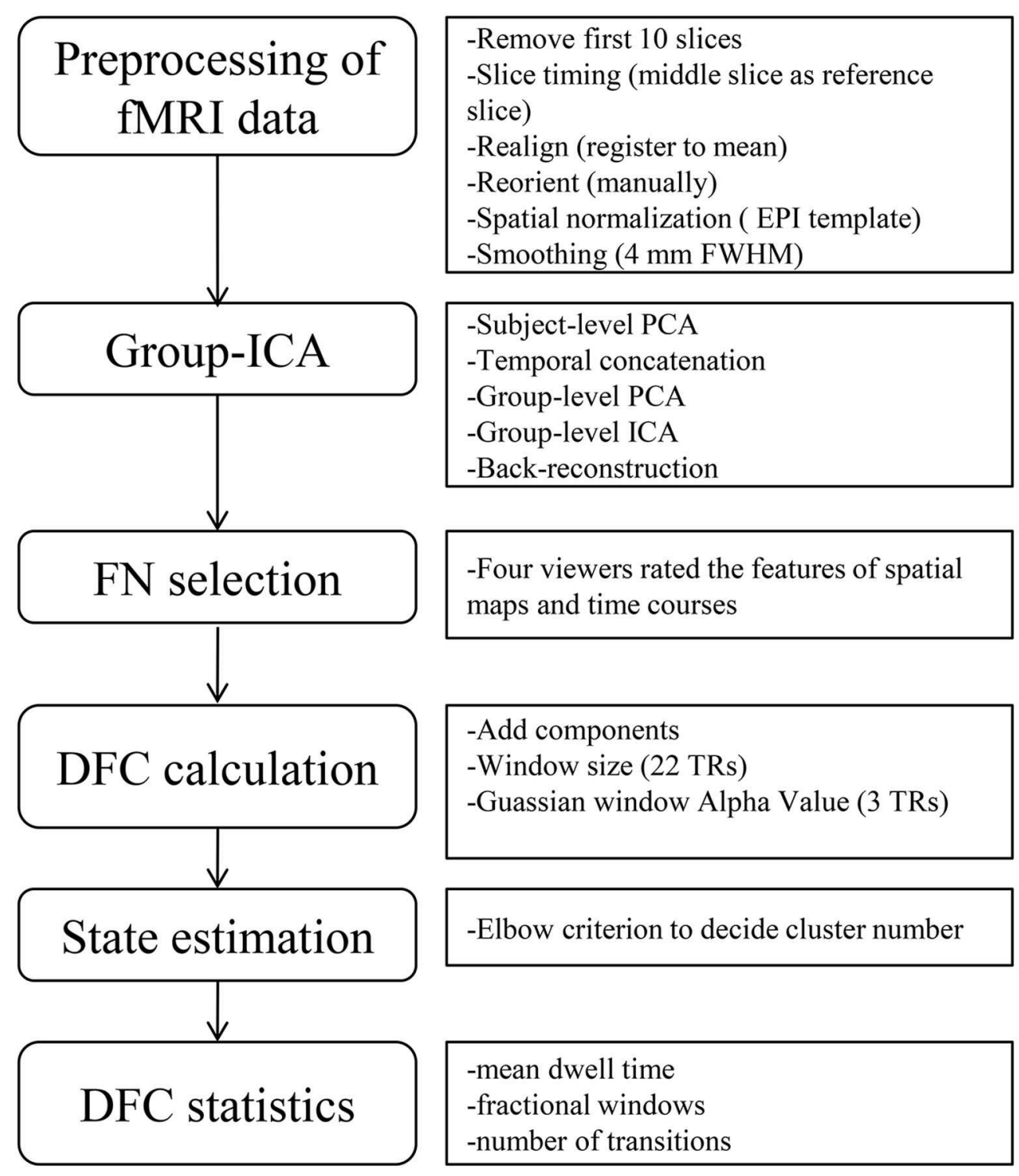

FIGURE 1 | Flow chart of data processing. The left part shows the overall steps of data processing, and the right part is the detailed sub-steps, including some important parameters in each step. ICA, independent component analysis; FN, functional networks; DFC, dynamic functional connectivity; FWHM, full width at half-maximum; PCA, principal component analysis; TRs, times of repetition.

replicate the decomposed independent components, the Infomax ICA algorithm was repeated for 20 times in ICASSO $(15,16)$ and the aggregate spatial maps were generated. With the group ICA back reconstruction method (17), the subject-specific spatial maps and their corresponding time courses were then generated.

\section{Functional Networks Selection}

To differentiate between resting-state functional networks (FN) and physiological components, a previously described procedure was applied (18). Four viewers visually inspected the spatial maps and average power spectra, and scores from 0 (definite artifact) to 1 (certain functional network) were given by them based on these expectations: (1) RSNs should exhibit peak activation in gray matter, low spatial overlap with vascular, ventricular, and susceptibility artifacts; (2) Time course should be dominated by low-frequency fluctuations, with ratio of the integral of spectral power below $0.10 \mathrm{~Hz}$ to the integral of power between 0.15 and $0.25 \mathrm{~Hz}$ (19). Following these criteria, components were divided into 3 categories: artifact (score $=0)$, mixed $(0<$ score $<3$ ), functional network (score $\geq 3$ ). This procedure resulted in 5 components located in 5 networks, default-mode (DMN), dorsal attention (DAN), auditory (AUD), sensorimotor (SMN), and visual (VN), shown in Figure 2.

Additional postprocessing of the selected components was performed following a previous research (6) to remove remaining noise sources. Briefly, steps included detrending, filtering with 


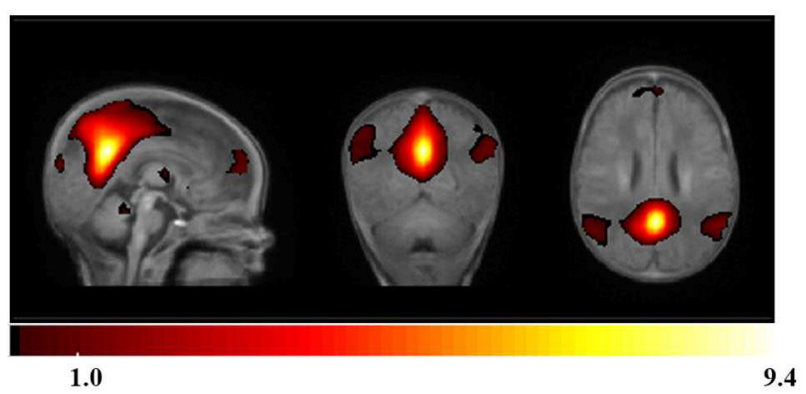

Peak Coodinates $(\mathrm{mm}) \quad(0,-39,21)$

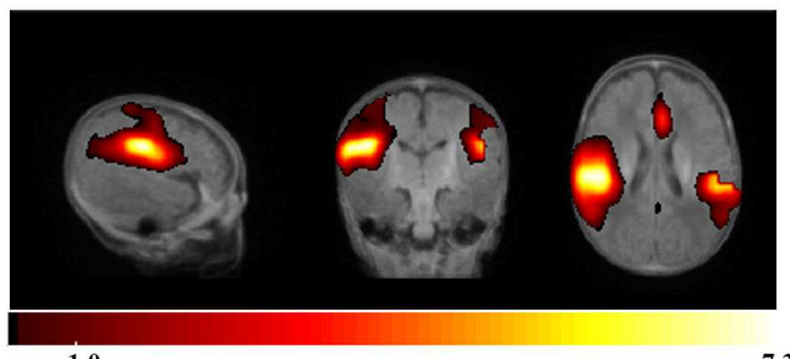

1.0

Peak Coodinates(mm) $(-33,-18,18)$

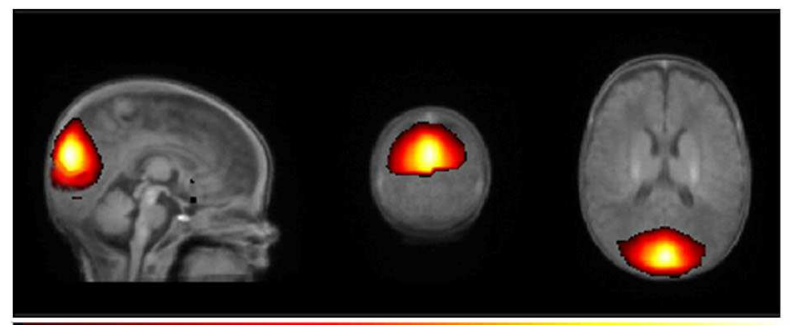

1.0 8.7

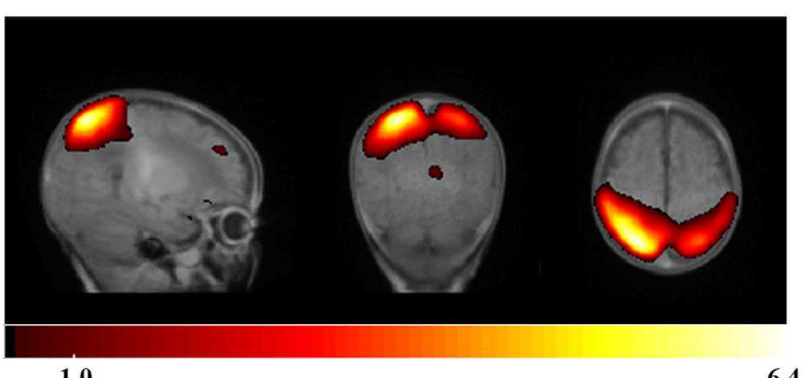

Peak Coodinates(mm) $(-18,-48,39)$

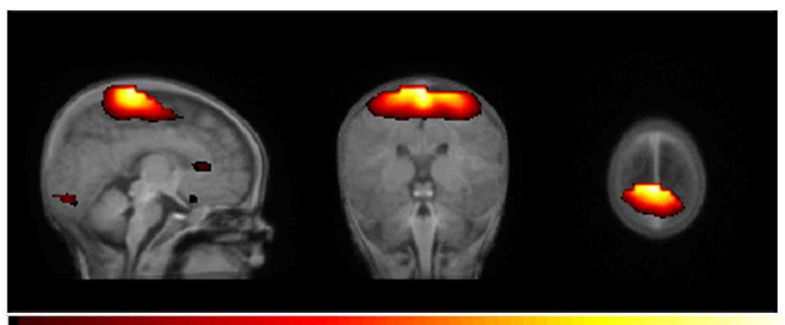

1.0

8.2

Peak Coodinates(mm) $(-2,-24,51)$

Peak Coodinates(mm) $(0,-63,15)$

FIGURE 2 | Identified networks. Based on the anatomical and functional properties, five components were located in five networks (DMN, DAN, AUD, SMN, VN). Brighter parts indicate stronger local activity, and under each part is its color bar and peak coordinates.

a cut-off value of low frequency fluctuation set as $0.15 \mathrm{~Hz}$, and despiking.

\section{DFC Analysis}

DFC was calculated based on a sliding window approach using GIFT toolbox. A sliding window length of 22 times of repetition (TRs) with a Gaussian window Alpha Value of 3 TRs was set, since this length could provide a good compromise between the quality of correlation matrix estimation and the ability to resolve dynamics (6). By sliding the window in a step length of 1 TR along the 230 TRs length scan, we obtained 208 consecutive windows across the entire scans. After computing DFC, all the functional connectivity matrices were transformed to $z$-score using Fisher's $z$-transformation for further analysis.

K-means method with sqEuclidean distance was used to regroup similar functional matrices of the different windows into different states, and the analysis was repeated 150 times to obtain a relatively stable result. The number of optimal clusters was calculated following the elbow criterion (6), and the number was set to be 4 .

We investigated the temporal properties of DFC states derived from the state vector of every infant. Three measures in subjects were assessed, including: (1) Mean dwell time, defined as the average number of consecutive windows belonging to one state before changing to the other state; (2) fractional windows, defined as the number of total windows belonging to one state; (3) number of transitions, defined as the number of times that the state switched from one to the other.

\section{Statistical Comparisons and Correlations Analysis}

The demographic and clinical data of all initially enrolled infants were recorded. Statistical analysis was performed 
TABLE 1 | Demographic and clinical data of all initial enrolled patients.

\begin{tabular}{|c|c|c|c|c|c|c|c|c|}
\hline & \multicolumn{2}{|c|}{ Preterm } & $t / x^{2}$ & $P$ & \multicolumn{2}{|c|}{ Term } & $t / x^{2}$ & $P$ \\
\hline Number & 34 & 63 & & & 37 & 31 & & \\
\hline Gestational age (weeks) & $35.93 \pm 1.34$ & $35.64 \pm 0.95$ & 1.11 & 0.27 & $40.29 \pm 1.06$ & $40.00 \pm 0.80$ & 1.04 & 0.30 \\
\hline Birth weight (kg) & $2.03 \pm 0.41$ & $2.03 \pm 0.23$ & -0.05 & 0.96 & $3.27 \pm 0.38$ & $3.29 \pm 0.39$ & -0.19 & 0.85 \\
\hline Twins or triplets & $2 / 32$ & $2 / 61$ & 0.41 & 0.61 & $2 / 35$ & $0 / 21$ & 1.18 & 0.53 \\
\hline Gender (male/female) & $15 / 19$ & $32 / 31$ & 0.39 & 0.67 & $19 / 18$ & $11 / 10$ & 0.00 & 1.00 \\
\hline Days of birth (days) & $9.47 \pm 2.02$ & $9.48 \pm 2.06$ & -0.01 & 0.99 & $9.62 \pm 2.30$ & $9.62 \pm 1.96$ & 0.00 & 1.00 \\
\hline PMA (weeks) & $37.28 \pm 1.34$ & $36.99 \pm 0.95$ & 1.10 & 0.28 & $41.66 \pm 1.13$ & $41.38 \pm 0.92$ & 0.96 & 0.34 \\
\hline \multicolumn{9}{|l|}{ Maternal factors } \\
\hline \multicolumn{9}{|l|}{ Clinical data after birth } \\
\hline Apgar-1min & $9.00 \pm 1.07$ & $9.24 \pm 1.00$ & -1.09 & 0.28 & $9.38 \pm 0.86$ & $9.48 \pm 0.51$ & -0.47 & 0.64 \\
\hline Apgar-5min & $9.68 \pm 0.48$ & $9.83 \pm 0.38$ & -1.57 & 0.12 & $10.00 \pm 0.00$ & $9.95 \pm 0.22$ & 1.00 & 0.32 \\
\hline Breast milk/mixed/formula & 1/23/10 & $1 / 40 / 22$ & 0.46 & 0.84 & $24 / 10 / 3$ & $12 / 8 / 1$ & 0.88 & 0.7 \\
\hline Ventilation time (hours) & $25.76 \pm 28.36$ & $15.81 \pm 25.67$ & 1.76 & 0.08 & $0.00 \pm 0.00$ & $2.29 \pm 10.47$ & -1.00 & 0.33 \\
\hline Apnea & $2 / 32$ & $2 / 61$ & 0.41 & 0.61 & 0 & 0 & & \\
\hline Sepsis & 0 & 0 & & & 0 & 0 & & \\
\hline Convulsions & 0 & 0 & & & 0 & 0 & & \\
\hline Hypothermia & $1 / 33$ & $1 / 62$ & 0.20 & 1.00 & 0 & 0 & & \\
\hline
\end{tabular}

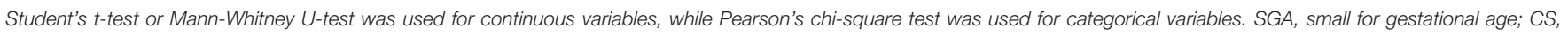

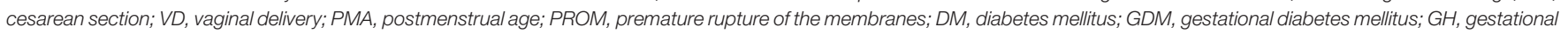
hypertension; PE, preeclampsia.

using Statistical Package for Social Science 24.0 (SPSS 24.0). Differences in the demographic and clinical data were compared between the final included and excluded infants, and differences in maturity and temporal properties of DFC were compared between the late preterm and term infants. For continuous variables, we used Student's $t$ test for parametric data and Mann-Whitney $U$-test for nonparametric data. Pearson's chi-square test was used to compare categorical variables. We also carried out Pearson's correlation analysis between altered temporal properties and indicators measuring the maturity (birth weight, gestational age, postmenstrual age) for all participants. Significance was set at a $P<0.05$ in all tests.

\section{RESULTS}

\section{Demographic and Clinical Characteristics}

Recorded characteristics of all initially recruited infants were compared between the final included and excluded infants, including demographic characteristics such as gestational age, days of birth, and postmenstrual age when scanned, some maternal factors during pregnancy, and clinical data during hospitalization. No significant differences were found between
TABLE 2 | Demographic characteristics.

\begin{tabular}{lcccc}
\hline & Late preterm & Term & $\boldsymbol{t} / \chi^{2}$ & $\boldsymbol{P}$-value \\
\hline Number & 34 & 37 & & \\
Gestational age (weeks) & $35.93 \pm 1.34$ & $40.29 \pm 1.06$ & -15.27 & $0.00^{*}$ \\
Days after birth (days) & $9.47 \pm 2.02$ & $9.62 \pm 2.30$ & -0.29 & 0.77 \\
Postmenstrual age (weeks) & $37.28 \pm 1.34$ & $41.66 \pm 1.13$ & -14.88 & $0.00^{\star}$ \\
Birth Weight (kg) & $2.03 \pm 0.41$ & $3.27 \pm 0.38$ & -13.38 & $0.00^{\star}$ \\
\hline
\end{tabular}

Student's t-test or Mann-Whitney U-test was used for continuous variables, while Pearson's chi-square test was used for categorical variables. Significant results are marked with $a^{*}$.

them, as shown in Table 1. These results indicated that characteristics of the final included and excluded infants were roughly balanced. Then we compared the indicators measuring maturity between final included late term and term infants, which were significantly different between groups, including gestational age (preterm:35.93 \pm 1.34 , term:40.29 $\pm 1.06 ; P$ $=0.00$ ), postmenstrual age when scanned (preterm: 37.28 \pm 1.34 , term:41.66 $\pm 1.13, P=0.00$ ), and birth weight (preterm: $2.03 \pm 0.41$, term: $3.27 \pm 0.38, P=0.00$ ), as shown in Table 2. 


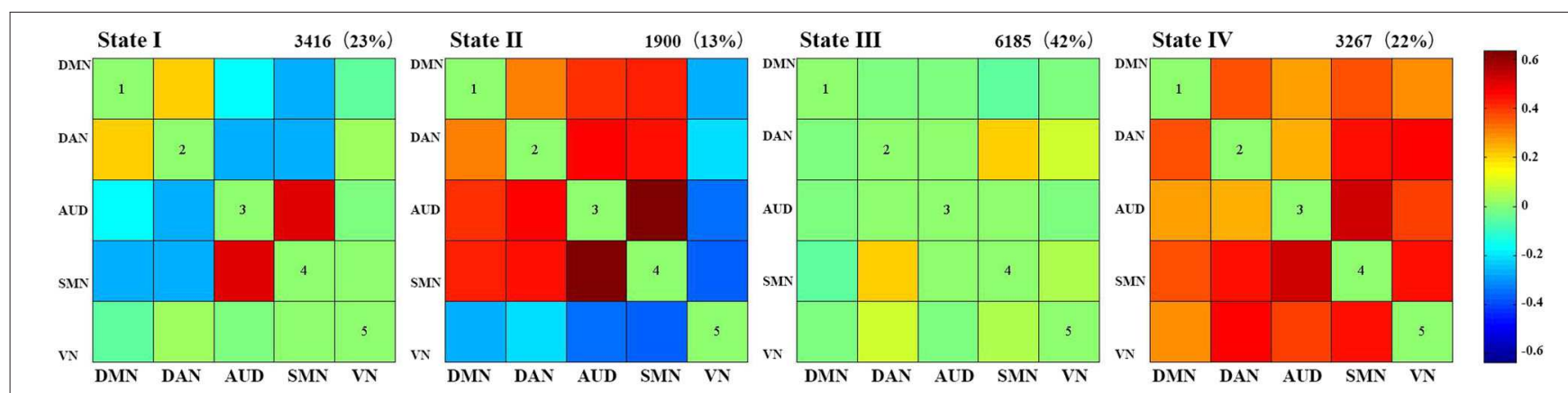

FIGURE 3 | Cluster centroids of each state. The total number of occurrences and percentage of total occurrences of each state are listed above each cluster. State I was dominated by weak connections except the connection between SMN and AUD, State II was characterized by relatively strong positive and negative connectivity, State III was characterized by general weak connectivity between networks, State IV demonstrated relatively strong positive connections across nearly all networks.

TABLE 3 | Temporal properties.

\begin{tabular}{|c|c|c|c|c|}
\hline & Preterm & Term & $t$ & $\boldsymbol{P}$ \\
\hline Dwell time (State I) & $10.36 \pm 12.67$ & $25.43 \pm 33.07$ & -2.49 & $0.02^{*}$ \\
\hline Dwell time (State II) & $14.83 \pm 15.96$ & $13.75 \pm 34.18$ & 0.17 & 0.87 \\
\hline Dwell time (State III) & $33.57 \pm 37.64$ & $18.50 \pm 11.71$ & 2.24 & $0.03^{*}$ \\
\hline Dwell time (State IV) & $22.12 \pm 35.76$ & $21.98 \pm 47.00$ & 0.01 & 0.99 \\
\hline Fractional windows (State I, \%) & $12.51 \pm 17.24$ & $32.89 \pm 26.26$ & -3.89 & $0.00^{*}$ \\
\hline Fractional windows (State II, \%) & $13.05 \pm 12.59$ & $12.69 \pm 20.56$ & 0.09 & 0.93 \\
\hline Fractional windows (State III, \%) & $48.11 \pm 25.69$ & $36.16 \pm 23.96$ & 2.02 & $0.04^{*}$ \\
\hline Fractional windows (State IV, \%) & $26.33 \pm 25.17$ & $18.26 \pm 26.34$ & 1.32 & 0.19 \\
\hline Number of transitions & $8.21 \pm 3.91$ & $8.57 \pm 4.32$ & -0.37 & 0.71 \\
\hline
\end{tabular}

For continuous variables, student's t-test was used for parametric data and Mann-Whitney U-test was used for non-parametric data, significant results are marked with a *

\section{Temporal Properties of Dynamic Connectivity in Each State}

We identified four reoccurring state patterns of functional connectivity based on the k-means clustering method. As illustrated in Figure 3, State III, which was characterized by general weak connectivity, occurred most frequently in all states (42\%), while State II, which was characterized by relatively strong positive and negative connectivity, occurred least frequently (13\%). More specifically, State III portrayed wide-spread weak between-network connectivity among all identified networks, including DMN, DAN, AUD, SMN, and VN, suggesting a rather static functional activity in State III. Additionally, State I occurred in $23 \%$ and State IV in $22 \%$ of all the windows. State IV demonstrated relatively strong positive connections across nearly all networks, whereas State I was dominated by weak connections except the connection between SMN and AUD.

As shown in Table 3, the mean dwell time in State I and State III was significantly different between groups. Specifically, the late preterm group showed a significantly shorter mean dwell time in State I (preterm: $10.36 \pm 12.67$, term: $25.43 \pm 33.07, P$ $=0.02$ ), while a significantly longer mean dwell time in state III (preterm: $33.57 \pm 37.64$, term: $18.50 \pm 11.71 ; P=0.03$ ). Notably, as shown in the violin plot in Figure 5B, some infants in the late preterm group spent extremely long time in State III, while no one in the term group did. As for the fractional windows, significant differences between groups were also identified in State I and State III. No significant group differences were identified in the mean dwell time or fractional windows in State II and State IV. The number of transitions was not significantly different between groups, either.

In summary, these changes indicated that the late preterm group switched states as frequently as the term infants, but they stay longer in State III than term infants. Since State III was characterized by general weak functional connectivity among all networks, it seemed that late preterm group preferred to stay in a more inactive state, compared with term neonates. Between-group comparison of cluster centroids of each state and the temporal properties of DFC were presented in Figures 4, 5 , respectively.

\section{Correlation Between Clinical Data and DFC Properties}

Correlation analysis was carried out to test whether the temporal properties of DFC were associated with the indicators measuring the maturity of included infants (gestational age, postmenstrual age, birth-weight). In line with our hypothesis, the mean dwell time and fractional time in State III showed a significant negative correlation with all these maturity indicators, whereas a positive correlation was found in state I, as shown in Table 4. 


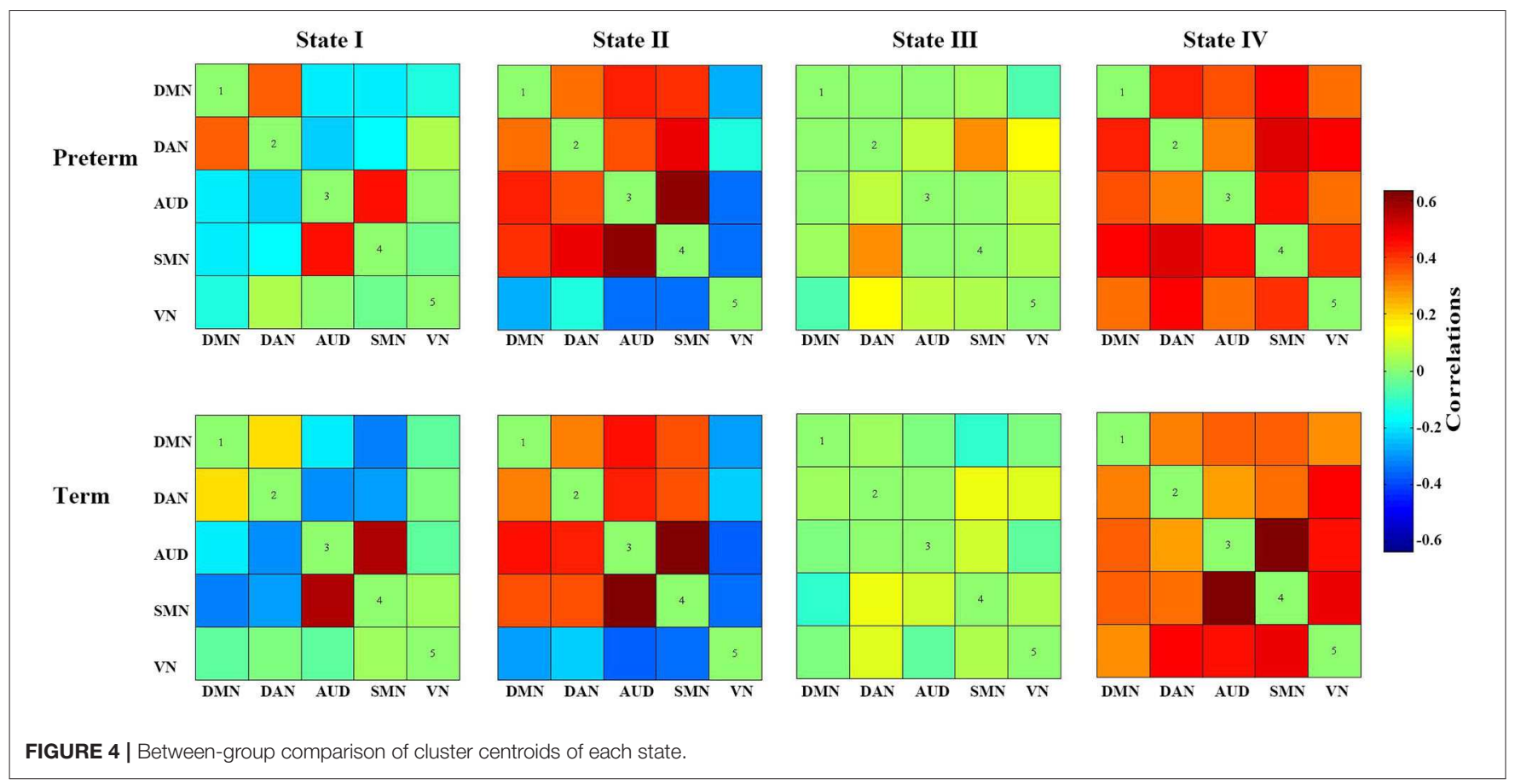

\section{DISCUSSION}

With increasing evidence emphasizing the neurodevelopmental problems faced by late preterm children (20), there is an increasing need to explore the possible underlying changes in their brains. Conventional brain MRI has the potential to detect even minor structural changes and help clinicians to make early diagnosis about premature brain injury. However, some late preterm infants without obvious structural changes also have developmental and emotional-behavioral problems in childhood and adolescence. Previous study has provided evidence for an aberrant structural and functional connectivity in preterm infants and a long-lasting impact of preterm birth on the organization of resting-state networks in school-aged children and adolescents $(21,22)$. Nonetheless, previous rs-fMRI studies of premature infants were mostly performed when they reached term equivalent age (23-25). As for the rapidly developing newborn brains, the best window for observing abnormalities may be missed. As far as we know, this is the first study that applied a DFC method to identify differences in the DFC properties between late preterm and term neonates, and the scanning time of all infants was from $34^{+6}$ to $43^{+2}$ weeks of postmenstrual age. Results demonstrated that a DFC approach can capture functional dynamics and reveal DFC characteristics in both term and late preterm brains across time.

In this study, we mainly focused on the temporal properties of the DFC, including the mean dwell time, fractional windows and the number of transitions. Besides, we investigated the association between these temporal properties and indicators measuring the maturity. Five networks (DMN, DAN, AUD, SMN, and $\mathrm{VN}$ ) were found in included neonates, which demonstrates a much simpler constitution than the functional networks in adults. Based on identified networks, four connectivity states were found across all participants. Significant differences existed between groups in the time staying in State III, which was characterized by general weak connectivity. The result indicated the preference for a weak connectivity state in late preterm infants. In addition, the correlation analysis showed the degree of maturation was negatively correlated to the dwell time and fractional time in state III, whereas state I showed the opposite result. The late preterm group spent less time in State I, which was dominated by weak connectivity but relatively strong connectivity between SMN and AUD. However, cautions are needed when explaining the result about State I, since it contains negative connections, and the retest reliability of negative connections was questioned by a previous research (26).

Since functional connectivity of premature infants of different gestational age demonstrates different development stages out of the uterus, it is worth noting that in both groups, SMN shows a strong connectivity with AUD in all states except State I, which is in line with the regular sequence of neurodevelopment of this period. Previous studies have identified multiple RSNs incorporating cortical and subcortical gray matter regions, including those located in primary motor and sensory cortices (e.g., SMN, VN, AUD) and those involving association cortices (e.g., DMN, DAN, frontoparietal control) $(23,27,28)$. It was reported that networks located in primary sensory and motor regions are established by term, and these networks demonstrate less variability between subjects (29). Our findings show that the connections between SMN and VN are strong in most of states in both late preterm and term group, which is consistent with the sequence of networks development identified by previous studies.

The causes and mechanisms of premature brain injury are so complex, so this study chose strict inclusion criteria to 

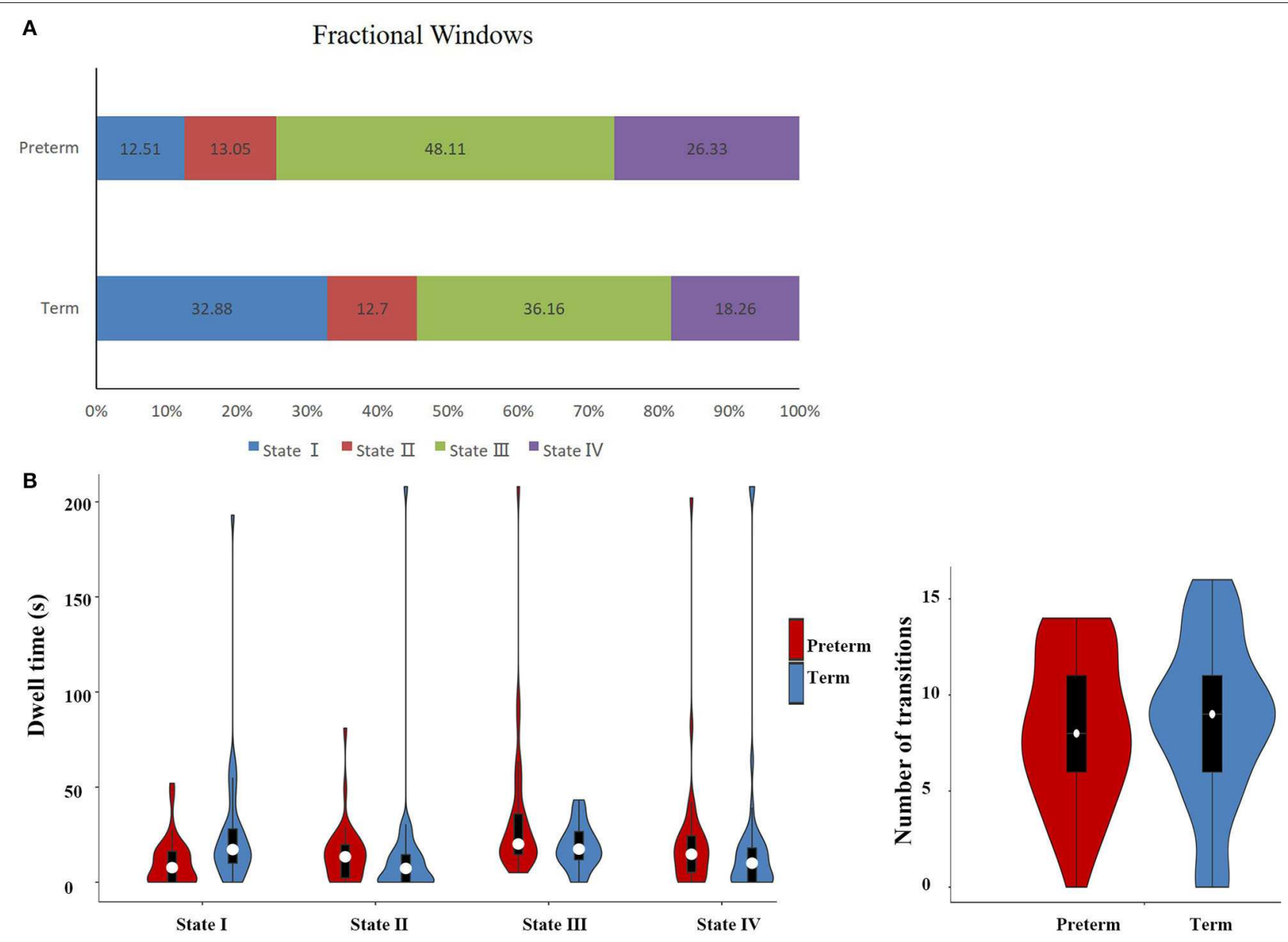

FIGURE 5 | Temporal properties of DFC states for the late preterm and term neonates. (A) Percentage of total time that all subjects spent in each state. (B) Mean dwell time and number of transitions between states were plotted using violin plots. The fractional windows and mean dwell time in State I and State III were significantly different between groups. It is worth noting that some infants in the late preterm group spent very long time in State III, while no one in the term group did.

make it possible to investigate one of the possible mechanisms. In addition, this study excluded infants with hemorrhage to simplify the research condition and facilitate the spatial normalization process. At the same time, this inclusion criteria may cause bias for the analysis, since preterm infants have a significantly higher chance of intracranial hemorrhage than term infants, which usually occurs within $72 \mathrm{~h}$ after birth and can lead to long-term neurological sequelae (30). Besides, a unique challenge was placed in image registration in infants, especially in those with brain injury. Specifically, due to the rapid changes in the size and cortical folding of brain in early life, notable heterogeneity exists in the registration process when we process data from neonates of different maturity. Our study included patients whose postmenstrual age is between $34^{+6}$ weeks and $43^{+2}$ weeks, and used the neonatal atlas for both term and late preterm infants. A narrower period for gestational-age specific atlas, such as 2-4 weeks, might make the spatial normalization more precise. In addition, we found some infants in the late preterm group spent extremely long time in State III, so the follow-up of neurodevelopment in these infants is very meaningful for they might have a higher chance of neurodevelopmental problems. But our study failed to perform reliable statistical analysis of the follow-up data due to the low completion rate of follow-up. However, though there is a lack of direct evidences to support the view that the length of time in State III is positively associated with neurodevelopment in the future, indirect evidences provided by previous studies of static functional connectivity indicate that preterm birth has persisting developmental effects on functional connectivity and motor performance in children, and altered static functional connectivity is related to motor development in childhood $(31,32)$. In addition, studies of DFC in adults have shown that the dwell time in a weakly connected state is associated with cognitive and intellectual impairment in a variety of neuropsychiatric disorders such as Parkinson's disease, autism and depression (33-35).

To conclude, this is the first study to assess dynamic connectivity properties in neonates. Compared to term neonates, the late preterm group shows a preference for 
TABLE 4 | Correlations between DFC temporal properties and clinical characteristics.

\begin{tabular}{|c|c|c|c|c|}
\hline & & GA & PMA & BW \\
\hline \multirow[t]{2}{*}{ Dwell time (State I) } & $r$ & 0.370 & 0.406 & 0.383 \\
\hline & $P$ & $0.001^{*}$ & $0.000^{*}$ & $0.001^{*}$ \\
\hline \multirow[t]{2}{*}{ Dwell time (State II) } & $r$ & -0.109 & -0.109 & -0.079 \\
\hline & $P$ & 0.367 & 0.367 & 0.511 \\
\hline \multirow[t]{2}{*}{ Dwell time (State III) } & $r$ & -0.338 & -0.327 & -0.292 \\
\hline & $P$ & $0.004^{\star}$ & $0.005^{\star}$ & $0.014^{*}$ \\
\hline \multirow[t]{2}{*}{ Dwell time (State IV) } & $r$ & 0.043 & 0.015 & -0.049 \\
\hline & $P$ & 0.724 & 0.898 & 0.685 \\
\hline \multirow[t]{2}{*}{ Fractional windows (State I, \%) } & $r$ & 0.529 & 0.558 & 0.508 \\
\hline & $P$ & $0.000^{\star}$ & $0.000^{\star}$ & $0.000^{*}$ \\
\hline \multirow[t]{2}{*}{ Fractional windows (State II, \%) } & $r$ & -0.114 & -0.120 & -0.059 \\
\hline & $P$ & 0.343 & 0.319 & 0.623 \\
\hline \multirow[t]{2}{*}{ Fractional windows (State III, \%) } & $r$ & -0.279 & -0.285 & -0.219 \\
\hline & $P$ & $0.018^{\star}$ & $0.016^{\star}$ & 0.067 \\
\hline \multirow[t]{2}{*}{ Fractional windows (State IV, \%) } & $r$ & -0.151 & -0.169 & -0.227 \\
\hline & $P$ & 0.209 & 0.158 & 0.057 \\
\hline \multirow[t]{2}{*}{ Number of transitions } & $r$ & 0.059 & 0.045 & 0.084 \\
\hline & $P$ & 0.627 & 0.712 & 0.485 \\
\hline
\end{tabular}

Pearson's correlation test was used, significant results are marked with a *. GA, gestational age; PMA, postmenstrual age; BW, birth-weight.

a state with general weak connectivity and the length of time in that state is negatively correlated to the degree of maturity. We believe these findings provide new perspectives for understanding the state-dependent neurophysiological mechanisms in premature brain injury. However, due to unique challenges associated with neonatal neuroimaging acquisition

\section{REFERENCES}

1. Blencowe H, Cousens S, Oestergaard MZ, Chou D, Moller AB, Narwal $\mathrm{R}$, et al. National, regional, and worldwide estimates of preterm birth rates in the year 2010 with time trends since 1990 for selected countries: a systematic analysis and implications. Lancet. (2012) 379:2162-72. doi: 10.1016/S0140-6736(12)60820-4

2. Salmaso N, Jablonska B, Scafidi J, Vaccarino FM, Gallo V. Neurobiology of premature brain injury. Nat Neurosci. (2014) 17:341-6. doi: 10.1038/nn.3604

3. Palumbi R, Peschechera A, Margari M, Craig F, Cristella A, Petruzzelli MG, et al. Neurodevelopmental and emotional-behavioral outcomes in latepreterm infants: an observational descriptive case study. BMC Pediatr. (2018) 18:318. doi: 10.1186/s12887-018-1293-6

4. Walsh BH, Inder TE. MRI as a biomarker for mild neonatal encephalopathy. Early Hum Dev. (2018) 120:75-9. doi: 10.1016/j.earlhumdev.2018.02.006

5. van den Heuvel MP, Sporns O. A cross-disorder connectome landscape of brain dysconnectivity. Nat Rev Neurosci. (2019) 20:435-46. doi: 10.1038/s41583-019-0177-6

6. Allen EA, Damaraju E, Plis SM, Erhardt EB, Eichele T, Calhoun VD. Tracking whole-brain connectivity dynamics in the resting state. Cereb Cortex. (2014) 24:663-76. doi: 10.1093/cercor/bhs352

7. Damaraju E, Allen EA, Belger A, Ford JM, McEwen S, Mathalon DH, et al. Dynamic functional connectivity analysis reveals transient states and analysis, further targeted studies were needed in this high-risk population.

\section{DATA AVAILABILITY STATEMENT}

The datasets generated for this study are available on request to the corresponding author.

\section{ETHICS STATEMENT}

The studies involving human participants were reviewed and approved by Ethics Committee of Daping Hospital, Army Medical University (Chongqing, China). Written informed consent to participate in this study was provided by the participants' legal guardian/next of kin.

\section{AUTHOR CONTRIBUTIONS}

YS and XM: study design, analysis, interpretation of data, drafting, and revising the article. XW: acquisition of data. YS final approval of the version to be published and providing funding for the study. All authors: contributed to the article and approved the submitted version.

\section{FUNDING}

This study was supported by National Natural Science Foundation of China (Grant No. 81741065 to YS).

\section{ACKNOWLEDGMENTS}

We are grateful to all of the study participants and their families for their cooperation.

of dysconnectivity in schizophrenia. Neuroimage Clin. (2014) 5:298-308. doi: 10.1016/j.nicl.2014.07.003

8. Yang Z, Craddock RC, Margulies DS, Yan CG, Milham MP. Common intrinsic connectivity states among posteromedial cortex subdivisions: insights from analysis of temporal dynamics. Neuroimage. (2014) 93(Pt 1):124-37. doi: 10.1016/j.neuroimage.2014.02.014

9. Wang J, Wang X, Xia M, Liao X, Evans A, He Y. GRETNA: a graph theoretical network analysis toolbox for imaging connectomics. Front Hum Neurosci. (2015) 9:386. doi: 10.3389/fnhum.2015.00458

10. Hutchison RM, Womelsdorf T, Allen EA, Bandettini PA, Calhoun $\mathrm{VD}$, Corbetta $\mathrm{M}$, et al. Dynamic functional connectivity: promise, issues, and interpretations. Neuroimage. (2013) 80:360-78. doi: 10.1016/j.neuroimage.2013.05.079

11. Power JD, Barnes KA, Snyder AZ, Schlaggar BL, Petersen SE. Spurious but systematic correlations in functional connectivity MRI networks arise from subject motion. Neuroimage. (2012) 59:2142-54. doi: 10.1016/j.neuroimage.2011.10.018

12. Shi F, Yap P-T, Wu G, Jia H, Gilmore JH, Lin W, et al. Infant brain atlases from neonates to 1- and 2-year-olds. PLoS ONE. (2011) 6:e18746. doi: 10.1371/journal.pone.0018746

13. Calhoun VD, Adali T, Pearlson GD, Pekar JJ. A method for making group inferences from functional MRI data using independent component analysis. Hum Brain Mapp. (2001) 14:140-51. doi: 10.1002/hbm.1048 
14. Erhardt EB, Rachakonda S, Bedrick EJ, Allen EA, Adali T, Calhoun VD. Comparison of multi-subject ICA methods for analysis of fMRI data. Hum Brain Mapp. (2011) 32:2075-95. doi: 10.1002/hbm.21170

15. Bell AJ, Sejnowski TJ. An information-maximization approach to blind separation and blind deconvolution. Neural Comput. (1995) 7:1129-59. doi: 10.1162/neco.1995.7.6.1129

16. Himberg J, Hyvärinen A, Esposito F. Validating the independent components of neuroimaging time series via clustering and visualization. NeuroImage. (2004) 22:1214-22. doi: 10.1016/j.neuroimage.2004.03.027

17. Calhoun VD, Adali T, Pearlson GD, Pekar JJ. Spatial and temporal independent component analysis of functional MRI data containing a pair of task-related waveforms. Hum Brain Mapp. (2001) 13:43-53. doi: 10.1002/hbm.1024

18. Allen EA, Erhardt EB, Damaraju E, Gruner W, Segall JM, Silva RF, et al. A baseline for the multivariate comparison of resting-state networks. Front Syst Neurosci. (2011) 5:2. doi: 10.3389/fnsys.2011.00002

19. Cordes D, Haughton VM, Arfanakis K, Wendt GJ, Turski PA, Moritz CH, et al. Mapping functionally related regions of brain with functional connectivity MR imaging. Am J Neuroradiol. (2000) 21:1636-44. Available online at: http:/ www.ajnr.org/content/ajnr/21/9/1636.full.pdf

20. Cheong JLY, Thompson DK, Olsen JE, Spittle AJ. Late preterm births: new insights from neonatal neuroimaging and neurobehaviour. Semin Fetal Neonatal Med. (2019) 24:60-5. doi: 10.1016/j.siny.2018.10.003

21. Rogers CE, Lean RE, Wheelock MD, Smyser CD. Aberrant structural and functional connectivity and neurodevelopmental impairment in preterm children. JNeurodev Disord. (2018) 10:38-38. doi: 10.1186/s11689-018-9253-x

22. Wehrle FM, Michels L, Guggenberger R, Huber R, Latal B, O'Gorman $\mathrm{RL}$, et al. Altered resting-state functional connectivity in children and adolescents born very preterm short title. NeuroImage Clin. (2018) 20:114856. doi: 10.1016/j.nicl.2018.10.002

23. Smyser CD, Snyder AZ, Shimony JS, Mitra A, Inder TE, Neil JJ. Resting-state network complexity and magnitude are reduced in prematurely born infants. Cereb Cortex. (2016) 26:322-33. doi: 10.1093/cercor/bhu251

24. Weinstein M, Ben-Sira L, Moran A, Berger I, Marom R, Geva R, et al. The motor and visual networks in preterm infants: an fMRI and DTI study. Brain Res. (2016) 1642:603-11. doi: 10.1016/j.brainres.2016.04.052

25. Tortora D, Severino M, Di Biase C, Malova M, Parodi A, Minghetti D, et al. Early pain exposure influences functional brain connectivity in very preterm neonates. Front Neurosci. (2019) 13:899. doi: 10.3389/fnins.2019.00899

26. Fox MD, Snyder AZ, Vincent JL, Corbetta M, Van Essen DC, Raichle ME. The human brain is intrinsically organized into dynamic, anticorrelated functional networks. Proc Natl Acad Sci USA. (2005) 102:9673-8. doi: 10.1073/pnas.0504136102
27. Smyser CD, Snyder AZ, Shimony JS, Blazey TM, Inder TE, Neil JJ. Effects of white matter injury on resting state fMRI measures in prematurely born infants. PLoS ONE. (2013) 8:e68098. doi: 10.1371/journal.pone.0068098

28. Smyser CD, Wheelock MD, Limbrick DD Jr, Neil JJ. Neonatal brain injury and aberrant connectivity. NeuroImage. (2019) 185:609-23. doi: 10.1016/j.neuroimage.2018.07.057

29. Gao W, Elton A, Zhu H, Alcauter S, Smith JK, Gilmore JH, et al. Intersubject variability of and genetic effects on the brain's functional connectivity during infancy. J Neurosci. (2014) 34:11288-96. doi: 10.1523/JNEUROSCI.5072-13.2014

30. Su B-H, Lin H-Y, Huang F-K, Tsai M-L, Huang Y-T. Circulatory management focusing on preventing intraventricular hemorrhage and pulmonary hemorrhage in preterm infants. Pediatr Neonatol. (2016) 57:45362. doi: 10.1016/j.pedneo.2016.01.001

31. Damaraju E, Phillips JR, Lowe JR, Ohls R, Calhoun VD, Caprihan A. Restingstate functional connectivity differences in premature children. Front Syst Neurosci. (2010) 4:23. doi: 10.3389/fnsys.2010.00023

32. Wheelock MD, Austin NC, Bora S, Eggebrecht AT, Melzer TR, Woodward LJ, et al. Altered functional network connectivity relates to motor development in children born very preterm. Neuroimage. (2018) 183:574-83. doi: 10.1016/j.neuroimage.2018.08.051

33. Díez-Cirarda M, Strafella AP, Kim J, Peña J, Ojeda N, Cabrera-Zubizarreta A, et al. Dynamic functional connectivity in Parkinson's disease patients with mild cognitive impairment and normal cognition. Neuroimage Clin. (2018) 17:847-55. doi: 10.1016/j.nicl.2017.12.013

34. Fiorenzato E, Strafella AP, Kim J, Schifano R, Weis L, Antonini A, et al. Dynamic functional connectivity changes associated with dementia in Parkinson's disease. Brain. (2019) 142:2860-72. doi: 10.1093/brain/awz192

35. Yao Z, Shi J, Zhang Z, Zheng W, Hu T, Li Y, et al. Altered dynamic functional connectivity in weakly-connected state in major depressive disorder. Clin Neurophysiol. (2019) 130:2096-104. doi: 10.1016/j.clinph.2019. 08.009

Conflict of Interest: The authors declare that the research was conducted in the absence of any commercial or financial relationships that could be construed as a potential conflict of interest.

Copyright (c) $2020 \mathrm{Ma}, \mathrm{Wu}$ and Shi. This is an open-access article distributed under the terms of the Creative Commons Attribution License (CC BY). The use, distribution or reproduction in other forums is permitted, provided the original author(s) and the copyright owner(s) are credited and that the original publication in this journal is cited, in accordance with accepted academic practice. No use, distribution or reproduction is permitted which does not comply with these terms. 